\title{
Semantic shot classification in soccer videos via playfield ratio and object size considerations.
}

\begin{abstract}
This paper presents a semantic shot classification algorithm for soccer videos. Generally, each shot within a match video is assigned either a far or close up-view class label. Initially, the playfield region for each frame within a shot is identified through low-level color image processing. An additional property is then considered namely the largest object size overlapping the playfield. Class labels are then accordingly assigned to each frame based on carefully constructed rule-sets. Majority voting is finally performed where the dominant frame labels within each shot is used as the ultimate class label. Experiments conducted on six soccer matches with varying camera shooting styles have been very promising, where the additional consideration of largest object size is able to significantly reduce the number of misclassifications.
\end{abstract}

Keyword: Shot classification; Scene classification; Soccer video analysis. 